\title{
Comparison of Top down and Bottom up Cost Approaches in Colon and Rectal Cancer Treatment
}

\author{
Rukiye Numanoğlu Tekin ${ }^{*}$, Bayram Şahin² \\ ${ }^{1}$ Baskent University, Ankara, Turkey \\ ${ }^{2}$ Hacettepe University, Ankara, Turkey \\ Email: ^rukiyenumanoglu@hotmail.com,numanoglu@baskent.edu.tr,baysahin@hacettepe.edu.tr
}

How to cite this paper: Tekin, R.N. and Şahin, B. (2021) Comparison of Top down and Bottom up Cost Approaches in Colon and Rectal Cancer Treatment. Health, 13, 90-109.

https://doi.org/10.4236/health.2021.132009

Received: November 24, 2020

Accepted: February 4, 2021

Published: February 7, 2021

Copyright (c) 2021 by author(s) and Scientific Research Publishing Inc.

This work is licensed under the Creative Commons Attribution-NonCommercial International License (CC BY-NC 4.0). http://creativecommons.org/licenses/by-nc/4.0/

\begin{abstract}
Introduction: In recent years, health care expenditures have significantly risen across the world and in Turkey. Because of the limited resources allocated to health, it is very important for countries to conduct studies especially on cost of diseases such as colorectal cancer (CRC) with a high burden of disease and labor loss. The aim of this study was to calculate the cost of treatment for colon and rectal cancers based on two different cost approaches from the perspective of SSI (top-down and bottom-up) and to compare the results obtained. Materials and Methods: Data were obtained from the SSI MEDULA System a total of 62,970 colon and rectal cancer patient for the top-down cost approach. In the bottom-up cost approach, treatment costs were determined based on the expert opinions. Results: The average cost of treatment per patient for colon cancer was determined as $3055.5 \mathrm{TL}$, for rectal cancer was determined as $4146.2 \mathrm{TL}$ with the top-down cost approach. The average cost of treatment per patient for colon cancer was determined as 14,920.4 TL, for rectal cancer was determined as 17,904.6 TL according to the bottom-up cost approach. Conclusion: Since the bottom-up cost approach provides more detailed information on the cost of illness, and thereby on the use of resources allocated to health, it is thought that the combined use of these two cost approaches completes each other's weaknesses.
\end{abstract}

\section{Keywords}

Cost of Illness, Colon Cancer, Rectal Cancer, Top Down Cost of Illness, Bottom Up Cost of Illness

\section{Introduction}

In recent years, health care expenditures have significantly risen across the world 
and in Turkey. Because of the limited resources allocated to health, it is very important for countries to conduct studies especially on cost of diseases such as colorectal cancer (CRC) with a high burden of disease and labor loss [1] [2] [3].

CRC is the third most common cancer type arising in any of the colon and rectum organs with over 1 million new cases annually. It is expected that the number of CRC cases will increase with aging population. Given the cancer-related deaths, it has been determined that about 600,000 people lose their lives every year due to CRC [1] [4] [5] [6]. In Turkey, as of the 2010 data, the incidence of CRC is 20.7 per 100 thousand in males and 13.1 per 100 thousand in females, and it has been found to be the third most common type of cancer in females, and the fourth most common type of cancer in males. According to the 2014 data of the Brief Report for Cancer Prevention and Screening in Turkey, 25 thousand men and women developled CRC within a year [7].

In general, there is a need for cost-of-illness studies to reveal the economic burden of diseases on the societal scale, thus enabling policy-makers and decision-makers to make future projections related to health expenditures and to take rational decisions on allocation of resources. As with other economic analyses, cost-of-illness studies are very challenging studies, and three types of costs are calculated in these studies: direct, indirect and intangible costs. Direct costs include healthcare costs for diagnosis, treatment and rehabilitation, and non-healthcare costs accrues as a result of conditions such as access to health services, household expenditure on health care, individuals changing their house, car or work due to a disease. Indirect costs refer to the productivity losses arising due to a disease. Costs caused by reasons such as work-day loss, decrease in productivity, change in individual's health condition, and decrease in the quality of life are calculated within indirect costs. Intangible costs refer to pain, anxiety, stress or other emotional problems caused by a disease and endured by the patient and their relatives, and the costs obtained here are generally presented as quality of life measures. The cost methodology to be selected for the calculation of costs substantially depends on the availability and accessibility of data, which varies considerably from one country to another [1] [8]-[22].

Many different approaches are used in cost-of-illness studies, and these approaches are categorized into three groups as the prevalence- and incidence-based approach, the top-down and bottom-up approach, and the retrospective and prospective approach. The prevalence-based approach involves the calculation of direct costs and productivity losses of all cases in the period of study of a disease or a group of diseases. Whereas, in the incidence-based approach, the lifetime costs of the disease-related conditions arisen in new cases within the determined study period are calculated. The main difference between these two methods is that the results obtained with the prevalence-based approach are generally greater than the results obtained from the incidence-based approach. This difference is usually due to the fact that the disease varies depending on whether or not it causes long-term sequelae. If the disease studied is not a disease causing 
long-term sequelae, there are no major differences between the costs calculated by these two approaches. The retrospective and prospective cost approaches are the approaches based on the temporal relationship between the onset of the study and the data collection. In the retrospective cost approach, all cases related to the study have shown up when the study started. This means that the data to be collected within the scope of the study are already registered data. Contrary to this, in the prospective cost approach, all cases related to the study have not yet come out when the study started, and it is necessary to follow up patients included in the study during the data collection phase [10] [11] [13] [21] [23]. The top-down and bottom-up cost-of-illness approaches, a third approach related to cost-of-illness approaches, stand out as commonly used methods in cost-of-illness studies which calculate the direct costs. The top-down cost method uses total health expenditures and disease-specific rates to demonstrate disease-specific costs. Costs are calculated by multiplying the total health expenditures by the utilization rate of health services by the disease group. Cost-of-disease studies using the top-down cost approach are more successful in demonstrating the problems in resource allocation. The bottom-up cost approach is based on the unit costs of the service provided. The cost calculation based on this method is performed in 2 steps. In the first step, the amount of health care inputs is determined, while the costs of the inputs used are determined in the second step, and the costs are calculated by multiplying the amount of health care inputs by the unit costs. In other words, the average cost of the provided service is calculated, and this calculated cost is adjusted to the total population receiving health care services so the total cost of health care service is found. The data on all resource consumption regarding the disease are obtained from the national health system in the top-down cost-of-illness studies, while the data are usually collected by using questionnaires or expert panel methods in the bottom up cost-of-illness studies [11] [13] [23] [24].

In the literature, there were 2 studies using the top-down and bottom-up cost approaches together. One of these is a study by Van Asselt et al. (2002) conducted in the Netherlands to determine the cost of Borderline Personality Disorder (BPD). One-year data of patients diagnosed with BPD was derived from the National Health Records of the Netherlands for the top-down cost approach. Whereas, a questionnaire was applied to $88 \mathrm{BPD}$ patients for the bottom-up cost approach. There were significant differences between the BPD costs obtained by the top-down and bottom-up cost approaches. The total yearly cost of BPD treatment was calculated as $200,184,828 €$ with the top-down cost approach, while the bottom-up cost approach was found to be 16 times higher than the top-down cost approach, and the total yearly cost of BPD treatment was calculated as $3,258,240,100 €$. This different result was explained by loss to follow-up patients. Accordingly, the amount of health service use determined by the bottom-up cost approach reflects the situation that BPD patients must comply with, while the amount of service obtained by the top-down cost approach reflects the 
real situation. It was demonstrated that patients who had BPD and did not adhere to the treatment and control processes had a significant effect on the costs calculated through the actual data. In the study by Koopmanschap et al. (2011) aimed to determine the cost of type 2 diabetes in the Netherlands using the top-down and bottom-up cost approaches, the national health records and reports were analyzed for the top-down cost approach, while the health records of 1.371 patients with type 2 diabetes were analyzed for the bottom-up cost approach. The cost of type 2 diabetes was calculated as 519 million euros using the top-down cost approach and as 567 million euros using the bottom-up cost approach, resulting in a $10 \%$ cost difference. In this study, it has been demonstrated that both methods are complementary to each other, and that the combined use of these approaches completes the weaknesses of the methods [25] [26].

Cost-of-illness studies using the top-down cost approach demonstrate the actual costs of a disease rather than an estimation. However, because of the fact that real patient data is required for these studies and the access to these data is often difficult and also, the data obtained does not contain detailed information on the use of health services, researchers tend towards the use of bottom-up cost approach in cost-of-illness studies. On the other hand, the bottom-up cost approach provides more detailed information on the cost of a disease, and thereby, on the use of resources allocated to health. With this cost approach, it is possible to obtain data based on the severity or stage of a specific disease. It is especially used as an important tool in determining the costs of diseases that are rare in the population or complex. In the studies using the bottom-up cost approach, instead of the actual disease data obtained from national health systems, a questionnaire is usually applied to the patients who are diagnosed with the relevant disease and specialist opinions are utulized, and thus the amount of health services use, which should be related to that disease, is demonstrated. Therefore, it is especially recommended that the bottom-up cost approach be used to efficiently plan the resources allocated to health services for the future. The national health data generally used in the top-down cost approach demonstrates the use of health services today, but remains incapable of making plan for the future. The reason for this is the behavioral differences of individuals in the case of illness and on the rational use of health services. Patients who were diagnosed with any disease but do not adhere to the treatment plan determined by the physician regarding this disease and do not have regular follow-ups are considered as "lost to follow-up" patients in the data obtained from the national health system, and these lost to follow-up patients cause the cost disease not to precisely reflect the reality. In the light of all these data, the cost of colon and rectal cancer was calculated using the top-down and bottom-up cost approaches together in this study, the costs obtained by these two approaches were compared. As a result of this comparison, the differences and similarities arisen in two different cost approaches were demonstrated and inferences were made to guide the future 
cost-of-ilness studies.

\section{Materials and Methods}

In order to determine treatment costs of colon and rectal cancer patients from SSI's perspective using the top down cost approach, we have used the MEDULA System. MEDULA Sytem was created by SSI in Turkey in order to collect invoice information electronically from health service providers and enable payment of services. Data from the MEDULA system were obtained in accordance with the ICD-10 code of the C18-Malignant Neoplasm of Colon and C20-Malignant Neoplasm of Rectum in excel file format 7 seperate folder. Data files were extracted into separate MS Access files to create a database, modifications necessary for analysis were made by using MS Access and MS SQL program later on, after which data were transferred to SPSS. Following such modifications, patient numbers from all files were matched with 62,970 patient data from the initial diagnosis file in order to extract data.

Based on data obtained from MEDULA system, we were able to gather information on age, sex, outpatient/inpatient admission numbers, the procedure/operation/treatment for outpatient/inpatient, laboratory and radiology tests conducted for outpatient/inpatient, services provided to inpatients at intensive care, drugs used in and prescribed by the hospital, comorbidity diseases, and length of stay.

In Turkey, the principles of payments made by the SSI for all services offered in health institutions are regulated by Health Application Notification (SUT). SUT is a legislative announcement which provides guidance, guiding, pricing, and other implementation details of the state's health-related social policies. SUT procedure scores of surgeries, laboratory and radiology examinations, clinical and intensive care administrations used in calculating medical costs have been calculated using SUT 2014 APP 2B-List of Procedure Scores per Service and APP 2C-List of Procedure Scores based on Diagnosis, with unit prices calculated from SSI's perspective. The list of drugs used was determined using the RX Media Program based on prices paid by the public in 2014 and demeaned to units used in the hospital for calculations. At the same time, prices of drugs prescribed were determined by using the RX Media Program based on prices paid by the public in 2014, which was multiplied by the amount of drugs prescribed to determine the total drug costs.

In the data obtained from the MEDULA system, the complications, metastasis and comorbidity data of the patients were not clearly defined. For this reason, complications and distant-near metastases that may occur during treatment are determined as follows according to expert opinions and information obtained from literature.

Complications:

- Bacterial intestinal infections

- Acute myocardial infaction 
- Pulmonary embolism

- Arterial embolism and thrombosis

- Bacterial and viral pneumonia

- Abscess of lung and mediastinum

- Noninfective gastroenteritis and colitis

- Ileus

- Haemorrhage of anüs and rectum

- Gastrointestinal haemorrhage

- Osteomyelitis

- Urinary tract infections

- Faecal incontinence

- Complications of surgery and medical care Metastasis:

- Malignant neoplasm of liver and intrahepatic bile ducts

- Malignant neoplasm of bronchus and lung

- Malignant neoğplasm of retroperitoneum and peritoneum

- Malignant neoplasm of brain

- Secondary malignant neoplasm of respiratory and digestive organs

- Secondary malignant neoplasm of other and unspecified sites

At the same time, patients with comorbidity were classified according to Charlson's Comorbidity Index (CCI) and treatment costs of colon and rectal cancer were calculated based on whether comorbidity is present. CCI has a score for each comorbidity and the scores of comorbidities in this study are as below:

- Diabetes Mellitus = 1 point

- Primary Hypertension $=1$ point

- Hypertensive Heart Disease $=1$ point

- Chronic Heart and Kidney Disease = 2 point

- Chronic Ischemic Heart Disease $=1$ point

- Chronic Obstructive Lung Disease $=1$ point

- Asthma = 1 point

In addition, metastatic diseases receive 6 points in this index. Based on the classification made according to total scores of patients, the intensity of comorbidities are mild for patients who scored 1 - 2, medium for patients who scored 3 - 4, and severe for patients who scored 5 and above; colon and rectal cancer medical costs were calculated using this classification.

In the bottom-up cost approach, in order to determine the treatment costs of colon and rectal cancers, a one-year treatment plan was developed and then the costs of treatment plans were calculated based on SUT. The treatment plan was developed in accordance with the information obtained from the literature and expert opinions. The one-year treatment plan was completed by 5 medical oncologists and 6 general surgery specialists, and surgical/medical treatment options and frequencies of colon and rectal cancer patients were determined according to expert opinions. 
The general surgeons' treatment plans included the following information:

- Percentage of patients diagnosed with colon and rectal cancer

- Distribution of patients according to disease stages (Stage I, II, III and IV)

- Hospitalization (Clinic and intensive care hospitalization and length of stay)

- Distribution of surgical operations according to colon and rectal cancer

- Post-surgical complications and frequencies

- Percentage of patient with metastasis

Medical oncologists' treatment plans included the following information according to disease stages:

- Frequencies of outpatient admissions

- Chemotherapy and radiotherapy treatments

- Hospitalization (Clinic and intensive care hospitalization and length of stay)

- Medical interventions during the hospitalization

- Examinations during the hospitalization (Laboratory, radiology, endoscopik, etc.)

- Medications

The forms filled by experts were entered into Microsoft Excel program and average values were obtained for each of the surgical and medical procedures performed for colon and rectum patients.

The data obtained with expert opinions were transferred to MS Excel program and mean values were obtained for each of the surgical and medical procedures performed for colon and rectal cancer patients. Then, the mean values were multiplied with SUT procedure scores and treatment costs of colon and rectal cancer patients were calculated.

In this study, treatment costs of colon and rectal cancer were displayed according to outpatient clinic, laboratory-radiology-patology, service and intensive care hospitalization, drugs and medical equipments, surgical intervention and complications.

We were not able to calculate teratment costs of colon and rectal cancer based on stages according to the top-down cost approach due to the fact that the data did not include disease stages. We were able to determine stage IV colon and rectal cancer patients in accordance with The Tumor-Node-Metastasis (TNM) classification only; we have compared medical costs of stage I-II-III patients and that of stage IV patients.

As the data obtained from the SSI MEDULA system did not include the first diagnosis dates of the patients, it could not be determined whether the patients included in the study were the newly diagnosed/active treatment patient or the follow-up patient (remission). On the other hand, in the bottom-up cost approach, it was determined that the experts did not take into account the follow-up patients. They accepted all patients as newly diagnosed/active treatment patients. However, it is known that medical costs of newly diagnosed/active teartment and follow-up patients differ significantly. In order to eliminate this difference between the two methods, expert opinions were used to determine 
newly diagnosed/active treatment patients and follow-up patients. According to expert opinions, patients who underwent colon and rectal cancer surgery in 2014 were identified as newly diagnosed patients and metastatic patients which determined according to TNM classification were accepted as active treatment patients. Colon and rectal cancer treatment costs were also determined based on data of these patients. Sensitivity analysis was performed by using Monte Carlo simulation method for the findings obtained from bottom-up cost approach.

\section{Results}

Of the 62,970 patients included in the study, 51,030 had colon cancer (81.0\%) and 11,940 had rectal cancer $(19.0 \%)$. The mean age of the patients with colon cancer was 61.2 years $( \pm 13.8)$ and the mean age of the patients with rectal cancer was 61.7 years $( \pm 13.2)$. Fifty-three percent of the colon cancer patients were male, while $56 \%$ of the rectal cancer patients were male. It was found that $49.2 \%$ of the colon cancer patients and $44.7 \%$ of the rectal cancer patients had comorbidities and the rate of primary hypertension was the highest among the comorbidities with $42.2 \%$ (Figure 1). According to the Charlson Comorbidity Index classification, it was demonstrated that of the colon cancer patients, $32.9 \%$ had mild, $6.4 \%$ had moderate and $17.1 \%$ had severe comorbidities, whereas, of the rectal cancer patients, $32.7 \%$ had mild, 5.3\% had moderate and $12.4 \%$ had severe comorbidities (Table 1). Given the medical or surgical treatment-related complication development, it was found that $38 \%$ of colon cancer patients and $40.2 \%$ of rectal cancer patients developed complications, and the rate of urinary tract infections was the highest with $32.6 \%$ (Figure 2). It was found that $16.9 \%$ of colon cancer patients and $12.2 \%$ of rectal cancer patients developed metastasis, and that malignant neoplasm of unspecified site showed up in $32 \%$ of the patients who developed metastasis (Figure 3 ).

In this study, the annual treatment costs for colon and rectal cancer patients were calculated by taking the outpatient admissions, admissions to the ward and intensive care unit, laboratory, imaging and pathological examinations carried out during admissions, drugs and materials used on and prescribed to patients, surgical interventions performed and costs of developed complications into

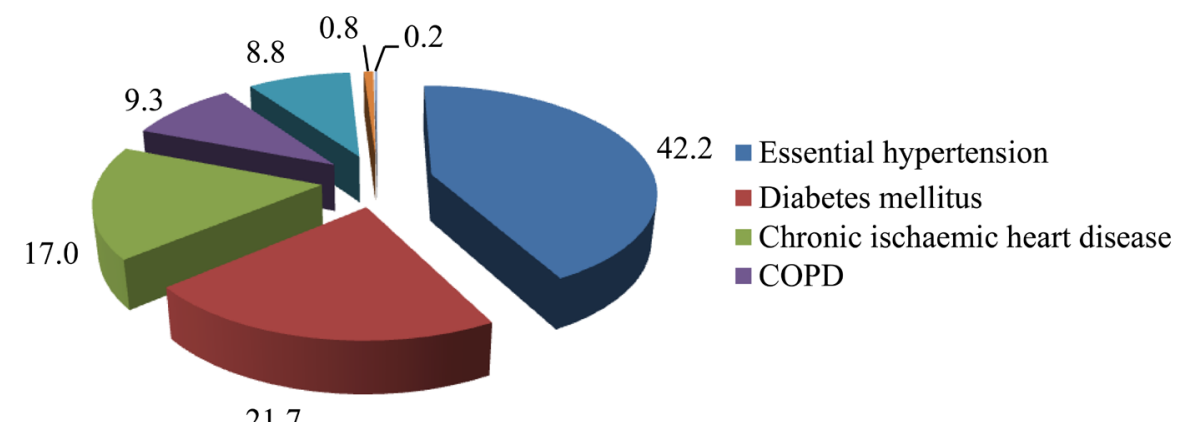

Figure 1. Distribution of Colon and Rectal Cancer Patients by Comorbidities, 2014, Turkey. 
Table 1. Summary statistics for colon and rectal cancer patients, 2014, Turkey.

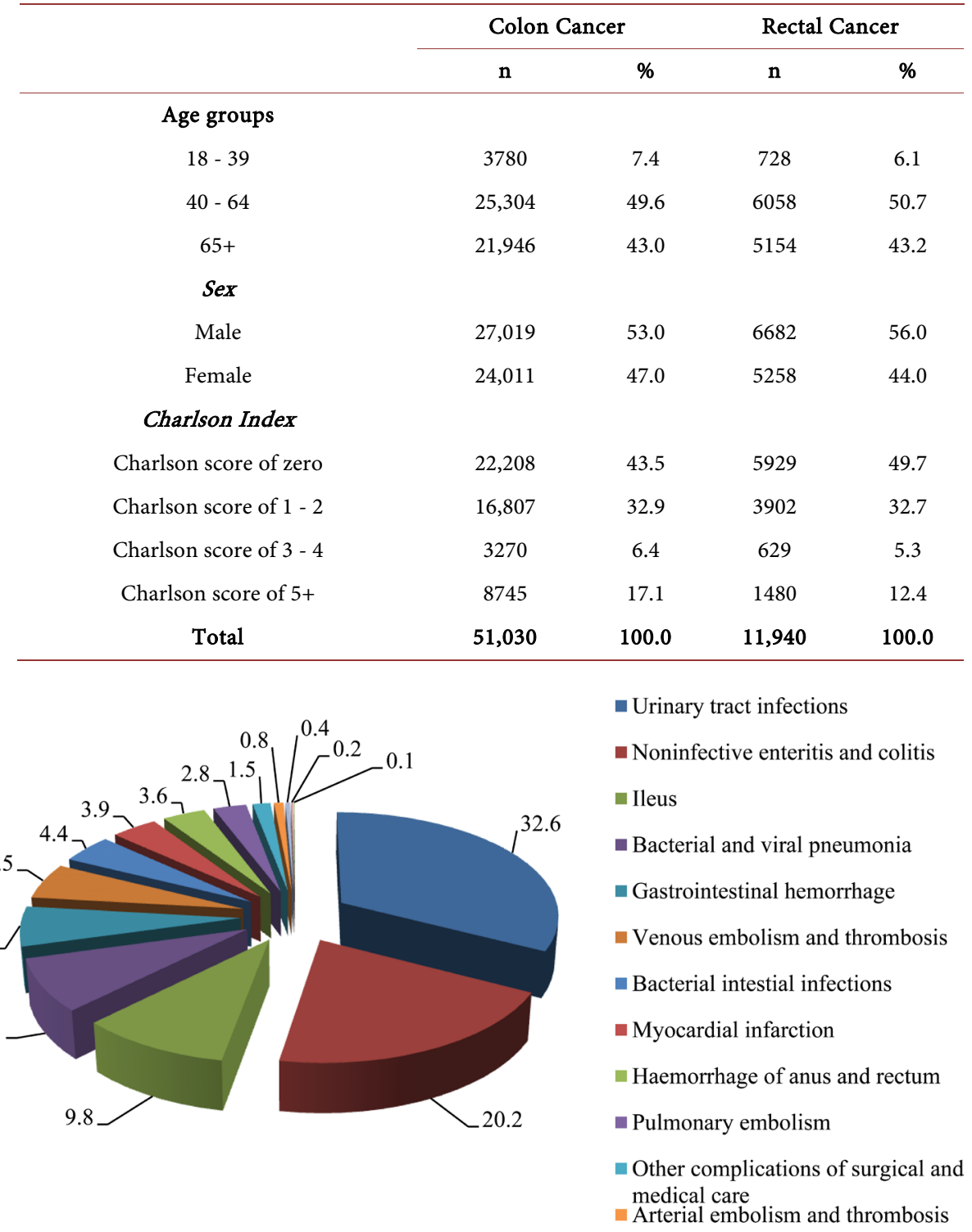

Figure 2. Distribution of Colon and Rectal Cancer Patients by Complications, 2014, Turkey.

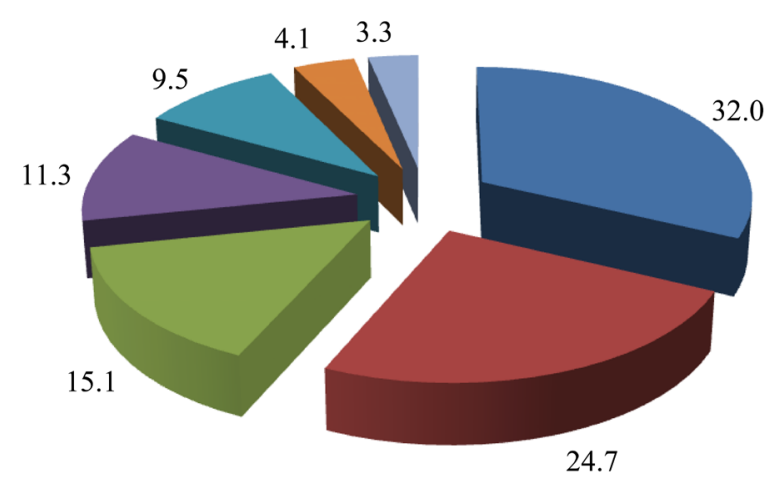

- Secondary malignant neoplasm of other and unspecified sites

- Malignant neoplasm of bronchus and lung

- Secondary malignant neoplasm of respiratory and digestive organs

- Malignant neoplasm of liver and intrahepatic bile ducts

- Secondary malignant neoplasm of other specified sites

Malignant neoplasm of retroperitoneum and peritoneum

- Malignant neoplasm of brain

Figure 3. Distribution of Colon and Rectal Cancer Patients by Metastasis, 2014, Turkey. 
account, since colon and rectal cancers are diseases in which polyclinic, clinical, intensive care and various laboratory services, as well as chemotherapy and various pharmacological agents and medical materials are used in the diagnosis and treatment stages, and the cost of complications encountered is also included.

As is seen in Table 2, the average cost of treatment per patient for colon cancer patients calculated using the top-down approach was determined as \$1393.7. Cosidering the distribution of the cost items, the highest average cost was for surgical intervention with $\$ 2305.1$, accounting for $31.9 \%$ of the total cost. The average cost of treatment per patient for rectal cancer patients calculated using the top-down approach was determined as $\$ 1891.2$. The highest average treatment cost was for surgical intervention with $\$ 2430.9$, accounting for $30.1 \%$ of the total cost.

In Table 3, the average cost of treatment per patient for colon cancer was determined as $\$ 6805.8$ according to the bottom-up cost approach. The average annual outpatient clinic cost was $\$ 366.1$, laboratory and imaging cost was $\$ 1645.9$, cost of admission to ward and intensive care unit was $\$ 698.3$, drug and material cost was $\$ 2307.0$, surgical intervention cost was $\$ 1375.6$ and complication cost was $\$ 412.9$. In Table 3, the average cost of treatment per patient calculated using the bottom-up approach was determined as $\$ 8167.0$ considering the staging rates in rectal cancer. The average annual outpatient clinic cost was $\$ 366.1$, laboratory and imaging cost was $\$ 1798.2$, cost of admission toward and

Table 2. Distribution of colon and rectal cancer treatment cost with top-down cost approach, 2014, Turkey.

\begin{tabular}{|c|c|c|c|c|}
\hline & $\mathrm{n}$ & Total (\$) & Per patient (\$) & $\%$ \\
\hline \multicolumn{5}{|l|}{ Colon Cancer } \\
\hline Outpatient & 45,480 & $864,035.4$ & 19.0 & 1.2 \\
\hline Laboratory-Radiology-Pathology & 50,267 & $10,767,544.1$ & 214.2 & 15.1 \\
\hline Inpatient & 15,127 & $22,346,800.3$ & 1477.3 & 31.4 \\
\hline Drugs and medical equipments & 39,879 & $11,505,503.3$ & 288.5 & 16.2 \\
\hline Surgical intervention & 9852 & $22,709,429.4$ & 2305.1 & 31.9 \\
\hline Complications & 19,380 & $2,928,057.5$ & 151.1 & 4.1 \\
\hline Total & 51,030 & $71,121,370.0$ & 1393.7 & 100.0 \\
\hline \multicolumn{5}{|l|}{ Rectal Cancer } \\
\hline Outpatient & 10,675 & $253,450.0$ & 23.7 & 1.1 \\
\hline Laboratory-Radiology-Pathology & 11,820 & $3,199,533.0$ & 270.7 & 14.2 \\
\hline Inpatient & 5239 & $8,551,143.1$ & 1632.2 & 37.9 \\
\hline Drugs and medical equipments & 9813 & $2,935,206.9$ & 299.1 & 13.0 \\
\hline Surgical intervention & 2792 & $6,787,133.9$ & 2430.9 & 30.1 \\
\hline Complications & 4794 & $854,808.0$ & 178.3 & 3.8 \\
\hline Total & 11,940 & $22,581,274.9$ & 1891.2 & 100.0 \\
\hline
\end{tabular}


Table 3. Distribution of colon and rectal cancer treatment cost with bottom-up cost approach, 2014, Turkey.

\begin{tabular}{ccccc}
\hline & Per patient (\$) & $\%$ & $\begin{array}{c}\text { Weighted per } \\
\text { patient }(\$)\end{array}$ & $\%$ \\
\hline Colon Cancer & & & & \\
Outpatient & 1282.7 & 4.7 & 366.1 & 5.4 \\
Laboratory-Radiology-Pathology & 6918.2 & 25.5 & 1645.9 & 24.2 \\
Inpatient & 2774.7 & 10.2 & 698.3 & 10.3 \\
Drugs and medical equipments & 8973.0 & 33.1 & 2307.0 & 33.9 \\
Surgical intervention & 5506.2 & 20.3 & 1375.6 & 20.2 \\
Complications & 1634.7 & 6.0 & 412.9 & 6.1 \\
Total & $27,089.5$ & 100.0 & 6805.8 & 100.0 \\
Rectal Cancer & & & & \\
Outpatient & 1282.7 & 4.2 & 366.1 & 4.5 \\
Laboratory-Radiology-Pathology & 7525.8 & 24.8 & 1798.2 & 22.0 \\
Inpatient & 2487.7 & 8.2 & 633.3 & 7.8 \\
Total & 9598.6 & 31.6 & 3004.3 & 36.8 \\
Drugs and medical equipments & 7823.2 & 25.8 & 1955.2 & 23.9 \\
Surgical intervention & 1639.0 & 5.4 & 409.7 & 5.0 \\
Complications & $30,357.0$ & 100.0 & 8167.0 & 100.0 \\
\hline & & & &
\end{tabular}

intensive care unit was $\$ 633.3$, drug and material cost was $\$ 3004.3$, surgical intervention cost was $\$ 1955.2$ and complication cost was $\$ 409.7$.

When the average cost of treatment per patient for metastatic and non-metastatic colon and rectal cancer patients taken from the MEDULA system was calculated according to the top-down cost approach, as is seen in Table 4, it was found that the treatment costs of metastatic colon and rectal cancer patients were higher than that of non-metastatic colon and rectal cancer patients. The average cost of treatment per patient for metastatic colon cancer patients was calculated as $\$ 2350.8$, while the average cost of treatment per patient for non-metastatic patients was calculated as $\$ 1199.4$. The average cost of treatment per patient for metastatic rectal cancer patients was calculated as $\$ 3018.8$, while the average cost of treatment per patient for non-metastatic patients was calculated as $\$ 1734$.3.

When the average cost of treatment per patient calculated using the bottom-up cost approach for patients with metastatic and non-metastatic colon and rectal cancer was analyzed, it was found that the treatment costs of patients with metastatic colon and rectal cancer were higher than that of patients with non-metastatic colon and rectal cancer. As is seen in Table 5, the average cost of treatment per patient for metastatic colon cancer patients was calculated as $\$ 13,578.9$ and the average cost of treatment per patient for non-metastatic patients was calculated $\$ 5112.4$. The average cost of treatment per patient for metastatic rectal cancer patients was calculated as $\$ 11,667.7$, while the average cost 
Table 4. Distribution of Metastatic (Stage IV) and Nonmetastatic (Stage I-II-II) Colon and Rectal Cancer Treatment Cost with Top-Down Cost Approach, 2014, Turkey.

\begin{tabular}{|c|c|c|c|c|c|c|}
\hline & \multicolumn{3}{|c|}{ Metastatic } & \multicolumn{3}{|c|}{ Nonmetastatic } \\
\hline & $\mathbf{n}$ & $\begin{array}{c}\text { Per } \\
\text { patient (\$) }\end{array}$ & $\%$ & $\mathbf{n}$ & $\begin{array}{c}\text { Per } \\
\text { patient (\$) }\end{array}$ & $\%$ \\
\hline \multicolumn{7}{|l|}{ Colon Cancer } \\
\hline Outpatient & 8031 & 22.9 & 1.0 & 37,449 & 18.2 & 1.5 \\
\hline Laboratory-Radiology-Pathology & 8512 & 336.7 & 14.3 & 41,755 & 189.2 & 15.8 \\
\hline Inpatient & 3172 & 1967.3 & 83.7 & 11,955 & 1347.2 & 112.3 \\
\hline Drugs and medical equipments & 8508 & 411.7 & 17.5 & 31,371 & 255.1 & 21.3 \\
\hline Surgical intervention & 783 & 2403.8 & 102.3 & 9069 & 2296.5 & 191.5 \\
\hline Complications & 4137 & 179.0 & 7.6 & 15,243 & 143.5 & 12.0 \\
\hline Total & 8614 & 2350.8 & 100.0 & 42,416 & 1199.4 & 100.0 \\
\hline \multicolumn{7}{|l|}{ Rectal Cancer } \\
\hline Outpatient & 961 & 31.2 & 1.0 & 9714 & 23.0 & 1.3 \\
\hline Laboratory-Radiology-Pathology & 1452 & 599.0 & 19.8 & 10,368 & 224.7 & 13.0 \\
\hline Inpatient & 852 & 2429.8 & 80.5 & 4387 & 1477.3 & 85.2 \\
\hline Drugs and medical equipments & 1438 & 457.8 & 15.2 & 8375 & 271.9 & 15.7 \\
\hline Surgical intervention & 381 & 2625.7 & 87.0 & 2411 & 2400.2 & 138.4 \\
\hline Complications & 752 & 183.5 & 6.1 & 4042 & 177.3 & 10.2 \\
\hline Total & 1459 & 3018.8 & 100.0 & 10,481 & 1734.3 & 100.0 \\
\hline
\end{tabular}

Table 5. Distribution of Metastatic (Stage IV) and Nonmetastatic (Stage I-II-II) Colon and Rectal Cancer Treatment Cost with Bottom-Up Cost Approach, 2014, Turkey.

\begin{tabular}{|c|c|c|c|c|}
\hline & \multicolumn{2}{|c|}{ Metastatic } & \multicolumn{2}{|c|}{ Nonmetastatic } \\
\hline & Per patient (\$) & $\%$ & Per patient (\$) & $\%$ \\
\hline \multicolumn{5}{|l|}{ Colon Cancer } \\
\hline Outpatient & 445.3 & 3.3 & 346.3 & 6.8 \\
\hline Laboratory-Radiology-Pathology & 4647.7 & 34.2 & 895.4 & 17.5 \\
\hline Inpatient & 1009.4 & 7.4 & 620.5 & 12.1 \\
\hline Drugs and medical equipments & 5582.2 & 41.1 & 1488.2 & 29.1 \\
\hline Surgical intervention & 1470.2 & 10.8 & 1351.9 & 26.4 \\
\hline Complications & 424.2 & 3.1 & 410.1 & 8.0 \\
\hline Total & $13,578.9$ & 100.0 & 5112.4 & 100.0 \\
\hline \multicolumn{5}{|l|}{ Rectal Cancer } \\
\hline Outpatient & 445.3 & 3.8 & 346.3 & 4.7 \\
\hline Laboratory-Radiology-Pathology & 4908.9 & 42.1 & 1020.5 & 14.0 \\
\hline Inpatient & 797.0 & 6.8 & 592.5 & 8.1 \\
\hline Drugs and medical equipments & 3061.8 & 26.2 & 2990.0 & 41.0 \\
\hline Surgical intervention & 2045.0 & 17.5 & 1932.7 & 26.5 \\
\hline Complications & 409.7 & 3.5 & 409.7 & 5.6 \\
\hline Total & $11,667.7$ & 100.0 & 7291.8 & 100.0 \\
\hline
\end{tabular}


of treatment per patient for non-metastatic patients was calculated as $\$ 7291.8$.

In line with the inclusion criteria, it was determined that of the 18,931 patients who were found to be active patients, 14,870 were colon and 4061 were rectal cancer patients, so the costs were recalculated. The average cost of treatment per patient for colon cancer patients was calculated as $\$ 3885.8$, while the average cost of treatment per patient for rectal cancer patients was calculated as $\$ 4644.6$ (Table 6).

\section{Results of Sensitivity Analysis}

A sensitivity analysis was carried out for the distribution and accuracy of the results obtained, and as a result of the sensitivity analysis, it was found that the 95\% confidence interval value for colon cancer treatment cost was between $\$ 2920.7$ - \$3017.8 $(\mathrm{n}=1000, \mathrm{X}=\$ 2971.3)$ for stage I, between $\$ 4222.5$ - \$4348.4 ( $\mathrm{n}$ $=1000, \mathrm{X}=\$ 4285.4)$ for stage II, between $\$ 5241.0-\$ 6942.4(\mathrm{n}=1000, \mathrm{X}=$ $\$ 6091.7)$ for stage III, and between $\$ 13,243.0-\$ 13,633.5(n=1000, X=\$ 13,438.3)$ for stage IV. Thus, the result of the sensitivity analysis confirmed the model created and the analysis results obtained (Table 7).

As a result of the sensitivity analysis, it was found that the $95 \%$ confidence interval value for rectal cancer treatment cost was between $\$ 3498.1$ - $\$ 3587.5$ ( $\mathrm{n}=$ $1000, X=\$ 3542.8)$ for stage I, between $\$ 5135.2$ - $\$ 5373.3$ for stage II $(n=1000$, $\mathrm{X}=\$ 5254.3)$, between $\$ 9296.5$ - \$9485.8 $(\mathrm{n}=1000, \mathrm{X}=\$ 9391.4)$ for stage III,

Table 6. Distribution of colon and rectal treatment cost with top-down cost approach according to newly diagnosed/active treatment or follow-up patients, 2014, Turkey.

\begin{tabular}{|c|c|c|c|c|}
\hline & $\mathbf{n}$ & Total (TL) & Per Patient (TL) & $\%$ \\
\hline \multicolumn{5}{|l|}{ Colon Cancer } \\
\hline Outpatient & 13,093 & $358,680.3$ & 27.4 & 0.6 \\
\hline Laboratory-Radiology-Pathology & 14,576 & $16,920,727.0$ & 1160.9 & 29.3 \\
\hline Inpatient & 8019 & $11,252.560 .7$ & 1403.2 & 19.5 \\
\hline Drugs and medical equipments & 11,285 & $5,218,504.1$ & 462.4 & 9.0 \\
\hline Surgical intervention & 9852 & $22,709,429.4$ & 2305.0 & 39.3 \\
\hline Complications & 7360 & $1,322,232.3$ & 179.7 & 2.3 \\
\hline Total & 14,870 & $57,782,133.9$ & 3885.8 & 100.0 \\
\hline \multicolumn{5}{|l|}{ Rectal Cancer } \\
\hline Outpatient & 3567 & $146,626.4$ & 41.1 & 0.8 \\
\hline Laboratory-Radiology-Pathology & 3897 & $5,328,066.0$ & 1367.2 & 28.2 \\
\hline Inpatient & 3054 & $4,413,664.1$ & 1445.2 & 23.4 \\
\hline Drugs and medical equipments & 3329 & $1,763,532.6$ & 529.8 & 9.3 \\
\hline Surgical intervention & 2792 & $6,787,133.9$ & 2430.9 & 36.0 \\
\hline Complications & 2195 & $422,618.7$ & 192.5 & 2.2 \\
\hline Total & 4061 & $18,861,641.7$ & 4644.6 & 100.0 \\
\hline
\end{tabular}


Table 7. Monte Carlo simulation results for total treatment cost of colon cancer patients.

\begin{tabular}{ccccc}
\hline & $\begin{array}{c}\text { Evre I } \\
\mathrm{n}=1000\end{array}$ & $\begin{array}{c}\text { Evre II } \\
\mathrm{n}=1000\end{array}$ & $\begin{array}{c}\text { Evre III } \\
\mathrm{n}=1000\end{array}$ & $\begin{array}{c}\text { Evre IV } \\
\mathrm{n}=1000\end{array}$ \\
\hline Mean & 2971.3 & 4285.4 & 6091.7 & $13,438.3$ \\
Median & 2688.0 & 3865.3 & 5451.8 & $13,645.8$ \\
Sd & 748.5 & 1015.4 & 1565.0 & 3149.2 \\
$\begin{array}{c}\text { \%95 Confidence } \\
\text { Interval }\end{array}$ & $2920.7-3017.8$ & $4222.5-4348.4$ & $5241.0-6942.4$ & $13,243.0-13,633.5$ \\
\hline
\end{tabular}

Table 8. Monte Carlo simulation results for total treatment cost of rectal cancer patients.

\begin{tabular}{ccccc}
\hline & $\begin{array}{c}\text { Evre I } \\
\mathrm{n}=1000\end{array}$ & $\begin{array}{c}\text { Evre II } \\
\mathrm{n}=1000\end{array}$ & $\begin{array}{c}\text { Evre III } \\
\mathrm{n}=1000\end{array}$ & $\begin{array}{c}\text { Evre IV } \\
\mathrm{n}=1000\end{array}$ \\
\hline Mean & 3542.8 & 5254.3 & 9391.4 & $11,445.9$ \\
Median & 3291.5 & 3906.4 & 9579.3 & $11,615.1$ \\
Sd & 72.1 & 192.0 & 1524.0 & 3578.9 \\
$\begin{array}{c}\text { \%95 Confidence } \\
\text { Interval }\end{array}$ & $3498.1-3587.5$ & $5135.2-5373.3$ & $9296.5-9485.8$ & $11,224.2-11,635.2$ \\
\hline
\end{tabular}

and between $\$ 11,224.2$ - $\$ 11,635.2$ ( $\mathrm{n}=1000, \mathrm{X}=\$ 11,445.9)$ for stage IV. Thus, the result of the sensitivity analysis confirmed the model created and the analysis results obtained (Table 8 ).

\section{Conclusions}

It was found that the incidence of CRC in Turkey from the year 2010 was 20.7 per 100 thousand in males and 13.1 per 100 thousand in females, in other words, it is the fourth most common type of cancer in males and the third most common type of cancer in females. These health problems, which are very important in terms of disease burden, are also thought to have a significant share in terms of the health resources they use; however, it is known that there is no study on the average treatment costs and cost components of both cancers. The aim of this study was to calculate the cost of treatment for colon and rectal cancers based on two different cost approaches from the perspective of SSI (top-down and bottom-up) and to compare the results obtained.

It was found that the average cost of treatment per patient for colon cancer patients calculated using the top-down cost approach was $\$ 1393.7$, while the average cost of treatment per patient calculated using the bottom-up cost approach was $\$ 6805.8$. It was found that the average cost of treatment per patient for rectal cancer patients calculated using the top-down cost approach was \$1891.2, while the average cost of treatment per patient calculated using the bottom-up cost approach was \$8167.0. According to these results, the cost of colon cancer treatment calculated using the bottom-up cost approach is 4.8 times higher than the cost of colon cancer treatment calculated using the top-down cost approach. In terms of the cost of rectal cancer treatment, the cost of treatment 
per patient calculated based on the bottom-up cost approach was 4.3 times higher than the cost of treatment per patient calculated using the top-down approach. One of the reasons for the difference between the costs of treatment per patient calculated based on two cost approaches may be due to the philosophy of the mentioned approaches. In the bottom-up cost approach, the cost of the services/resources that need to be used by the patients within a year based on specialist opinions and their unit costs were taken into consideration (i.e., the cost is calculated through what should be calculated), whereas in the top-down cost approach, the cost was calculated using the actual patient data taken from the MEDULA system. Therefore, since patients who were diagnosed with colon and rectal cancer but do not adhere to the treatment plan recommended by the physician and do not have regular follow-ups will not be seen in the MEDULA system, the treatment costs may be higher in the top-down cost approach. Another reason for the cost difference between the two methods is that since stage I colon and rectal cancer patients do not use any drug, are not treated and the majority of them are followed by family physicians, the services provided to these patients by family physicians are not included in the MEDULA system, and thus, the treatment cost may be lower in the top-down cost approach. Another reason for the cost difference is thought to be caused by drugs used in the treatment of colon and rectal cancer. It is known that chemotherapy drugs used in both cancers are costly and chemotherapy drugs that could be prescribed in 2014 were limited. As a result of the interviews conducted with the specialists, it was learnt that numerous drugs have been used other than the drugs that could be prescribed and these drugs are brought by means of Turkey Pharmacists' Association (TPA) and included in repayment cover; however, since the information on drugs brought by means of TPA is not entered the MEDULA system, it is thought that the treatment cost calculated based on the top-down cost approach may be lower. Moreover, it is thought that the conditions of the hospitals where physicians work also affected the treatment costs of colon and rectal cancer calculated using the bottom-up cost approach. In the data obtained from the specialist opinions, it was noted that some physicians stated that there was a shortage of hospital beds in the hospital where they work, and therefore, some of the inpatient services had to be provided outpatiently or the exact opposite conditions occurred. In the results obtained from the specialist opinions for the bottom-up cost approach, another point is that all patients are considered as active patients and the patients in remission are not taken into consideration. This caused the costs to be higher in the bottom-up cost approach. Since the first diagnosis dates of patients are not present in the actual patient data, whether the patient was newly or previously diagnosed could not be distinguished; however, because of the fact that the patients both receiving active treatment and patients in remission are present in the actual patient data, lower costs were evaluated to be an expected result. In order to eliminate this difference between the two methods, the specialists were re-interviewed and the study inclusion criteria 
were set to classify active patients and patients in remission included in the study in the data obtained from the SSI MEDULA system, and in the direction of these criteria, it was determined that there were 18,931 active patients, 14,870 colon and 4061 rectal cancer patients, and the costs were recalculated. As a result of this calculation, the average cost of treatment per patient for colon cancer patients was calculated as $\$ 3885.8$ and the average cost of treatment per patient for rectal cancer patients was calculated as $\$ 4644.6$. As a result of this calculation in line with the inclusion criteria, the cost of colon cancer treatment calculated using the bottom-up cost approach was 1.8 times higher than the cost of colon cancer treatment calculated using the top-down cost approach and also 1.8 times higher than the cost of rectal cancer treatment.

In this study, it was determined that higher cost of the disease calculated using the bottom-up cost approach than the cost of disease calculated using the top-down cost approach is consistent with the studies in the literature. There was no study using these two cost methods together in the calculation of the cost of treatment for colon and rectal cancer, there were only two studies used these two approaches together. One of these studies is a study by Van Asselt et al. aimed to determine the cost of Borderline Personality Disorder (BPD) conducted in the Netherlands, the total annual cost of BDP treatment was calculated as $200,184,828 €$ using the top-down cost approach, while the bottom-up cost approach was found to be approximately 16 times higher than the top-down cost approach and the total annual cost of BDP treatment was calculated as 3,258,240,100 €. This different result was explained by loss to follow-up patients. Accordingly, the amount of health service use determined by the bottom-up cost approach reflects the situation that BPD patients must comply with, while the amount of service obtained by the top-down cost approach reflects the real situation. It was demonstrated that patients who had BPD and did not adhere to the treatment and control processes had a significant effect on the costs calculated through the actual data. The other study was conducted by Koopmanschap et al. (2011) to determine the cost of type 2 diabetes in the Netherlands, and the cost of type 2 diabetes was calculated as 519 million euros using the top-down cost approach and 567 million euros using the bottom-up approach, and a cost difference of $10 \%$ has emerged. In this study, it has been demonstrated that both methods are complementary to each other, and that the combined use of these approaches completes the weaknesses of the methods. Two different studies were conducted based on different cost approaches to determine the cost of CRC treatment in Germany in 2007. One of these was conducted by Neubauer and Minartz (2007) based on the prevalence using the top-down cost approach and the average cost of CRC treatment was calculated as $21,820 €$. In another study by Sieg and Brenner (2007), the treatment costs of CRC patients were determined in line with the specialist opinions using the bottom-up cost approach, and the average cost of CRC treatment was calculated as $49,563 €[25][26][27][28]$. 
In the calculations using the top-down cost approach, the average cost of treatment per patient for metastatic colon cancer patients was calculated as $\$ 2350.8$, while the average cost of treatment per patient for non-metastatic patients was calculated as $\$ 1199.4$. The average cost of treatment per patient for metastatic rectal cancer patients was calculated as $\$ 3018.8$, while the average cost of treatment per patient for non-metastatic patients was calculated as $\$ 1734.3$ TL. In the calculations using the bottom-up cost approach, the average cost of treatment per patient for metastatic colon cancer patients was calculated as $\$ 13,578.9$, while the average cost of treatment per patient for non-metastatic patients was calculated $\$ 5112.4$. The average cost of treatment per patient for metastatic rectal cancer patients was calculated as $\$ 11,667.7$, while the average cost of treatment per patient for non-metastatic patients was calculated as $\$ 7291.8$. When the results obtained from both cost approaches according to the stages are analyzed, it can be said that the cost incurred for the treatment of the disease increases as the disease progresses, and this result is also consistent with the results of the studies in the literature. In a study by Haug et al. (2014) on the cost of CRC treatment in Germany, the average annual cost of CRC treatment was calculated as $29,400 €$ for stage I, $6100 €$ for stage II-III, and $64,600 €$ for stage IV. In the same study, the costs of colon and rectal cancer were calculated separately, and it was demonstrated that the cost of treatment for rectal cancer was higher than the cost of treatment for colon cancer. In a study by Chen et al. (2015) on the cost of treatment for colon adenocarcinomas conducted in Taiwan using the top-down cost approach, the data of 17,526 patients were collected from the Taiwan cancer records between 2002 and 2009, and the average lifetime treatment cost for colon adenocarcinomas was calculated as $\$ 8416$ for stage II, $\$ 14,334$ for stage III, and $\$ 21,837$ for stage IV [5].

The problems in accessing the actual data still persist in many countries and Turkey. In order to be able to support the decision-making processes of the health policy-makers by scientific studies, it is of great importance to conduct correct and unbiased studies. The conclusion of this study is that the results based on specialist opinions are reliable according to the sensitivity analysis carried out. In addition, it has been demonstrated that it is possible to obtain data based on the severity of a certain disease using the bottom-up cost approach, and it can therefore be proposed as a tool that can be used to determine especially the costs of rare or complex diseases in the community.

Cost-of-illness studies conducted using the top-down cost approach are generally based on data obtained from the national health system, and as is seen in the present study, the fact that the data in the SSI MEDULA system does not include detailed information on the use of health services leads to a lack of results obtained from the cost-of-illness studies. Since the bottom-up cost approach provides more detailed information on the cost of illness, and thereby on the use of resources allocated to health, it is thought that the combined use of these two cost approaches completes each other's weaknesses. For this reason, it is rec- 
ommended to demonstrate the cost of the same disease using the bottom-up cost approach in the cost-of-illness studies conducted with the actual disease data obtained from the national health system.

This research did not receive any specific grant from funding agencies in the public, commercial, or not-for-profit sectors.

This research does not contain any studies with human participants or animals performed by any of the authors. Therefore, ethical approval and informed consent does not require. The only permission was received by SSI to use data.

\section{Acknowledgments}

No one else contributed to the preparation of the manuscript.

\section{Conflicts of Interest}

The authors declare no conflicts of interest regarding the publication of this paper.

\section{References}

[1] Kriza, C., Emmert, M., Wahlster, P., Niederlander, C. and Kolominsky-Rabas, P. (2013) Cost of Illness in Colorectal Cancer: An International Review. Pharmacoeconomics, 31, 577-588. https://doi.org/10.1007/s40273-013-0055-4

[2] Keshavarz, K., Kebriaeezadeh, A., Alavian, S.M., Sari, A.A., Dorkoosh, F.A., Keshvari, M., Malekhosseini, S.A., Nikeghbalian, S. and Nikfar, S. (2015) Economic Burden of Hepatitis B Virus-Related Diseases: Evidence from Iran. Hepatitis Monthly, 15, e25854. https://doi.org/10.5812/hepatmon.15(4)2015.25854

[3] Ceilleachair, A., Costello, L., Finn, C., Timmons, A., Fitzpatrick, P., Kapur, K., Staines, A. and Sharp, L. (2012) Inter-Relationships between the Economic and Emotional Consequences of Colorectal Cancer for Patients and Their Families: A Qualitative Study. BMC Gastroenterology, 12, 62-72.

https://doi.org/10.1186/1471-230X-12-62

[4] Ceilleachair, A., Hanly, P., Skally, M., O’Neill, C., Fitzpatrick, P., Kapur, K., Staines, A. and Sharp, L. (2013) Cost Comparisons and Methodological Heterogeneity in Cost-of-Illness Studies: The Example of Colorectal Cancer. Medical Care, 51, 339-350. https://doi.org/10.1097/MLR.0b013e3182726c13

[5] Haug, U., Engel, S., Verheyen, F. and Linder, R. (2014) Estimating Colorectal Cancer Treatment Costs: A Pragmatic Approach Exemplified by Health Insurance Data from Germany. PLoS ONE, 9, e88407.

https://doi.org/10.1371/journal.pone.0088407

[6] Huang, W., Liu, G., Zhang, X., Fu, W., Zheng, S., Wu, Q., Liu, C., Liu, Y., Cai, S. and Huang, Y. (2014) Cost-Effectiveness of Colorectal Cancer Screening Protocols in Urban Chinese Populations. PLoS ONE, 9, e109150. https://doi.org/10.1371/journal.pone.0109150

[7] Ministry of Health (2014) Kolorektal Kanser Taramaları Tarama Programları. https://hsgm.saglik.gov.tr/depo/birimler/kanser-db/yayinlar/raporlar/kolorektal.pdf

[8] Cooper, B.S. and Rice, D.P. (1976) The Economic Cost of Illness Revisited. Social Security Bulletin, 39, 21-36.

[9] Hodgson, T.A. and Meiners, M.R. (1982) Cost-of-Illness Methodology: A Guide to 
Current Practices and Procedures. Health and Society, 60, 429-462. https://doi.org/10.2307/3349801

[10] McIntosh, E. (1996) The Cost of Rheumatoid Arthritis. British Journal of Rheumatology, 35, 781-790. https://doi.org/10.1093/rheumatology/35.8.781

[11] Songer, T.J. and Ettaro, L. (1998) Studies on the Cost of Diabetes. http://www.pitt.edu/ tjs/coi/Costofillness.PDF

[12] Segel, J.E. (2006) Cost-of-Illness Studies-A Primer. RTI International RTI-UNC Center of Excellence in Health Promotion Economics. https://pdfs.semanticscholar.org/3bbf/0a03079715556ad816a25ae9bf232b45f2e6.pdf

[13] Tarricone, R. (2006) Cost-of-Illness Analysis. What Room in Health Economics? Health Policy, 77, 51-63. https://doi.org/10.1016/j.healthpol.2005.07.016

[14] Konnopka, A., Leichsenring, F., Leibing, E. and König, H.H. (2009) Cost-of-Illness Studies and Cost-Effectiveness Analyses in Anxiety Disorders: A Systematic Review. Journal of Affective Disorders, 114, 14-31. https://doi.org/10.1016/j.jad.2008.07.014

[15] Sam, K.G., Kuriachan, M.A. and Philip, S. (2009) Pharmacoeconomics: Cost of Illness Studies. HYGEIA Journal of Drugs and Medicines, 1, 46-49.

[16] Tatar, M. and Wertheimer, A.I. (2010) Sağlık Teknolojilerinin Değerlendirilmesi: İlaç Geri Ödeme Kararları için Bir Model Önerisi. MN Medikal \& Nobel, Ankara.

[17] Costa, N., Derumeaux, H., Rapp, T., Garnault, V., Ferlicoq, L., Gilette, S., Andrieu, S., Vellas, B., Lamure, M., Grand, A. and Molinier, L. (2012) Methodological Considerations in Cost of Illness Studies on Alzheimer Disease. Health Economics Review, 2, 18. https://doi.org/10.1186/2191-1991-2-18

[18] Bendeck, M., Serrano-Blanco, A., Garcia-Alonso, C., Bonet, P., Jorda, E., Sabes-Figuera, R. and Salvador-Carulla, L. (2013) An Integrative Cross-Design Synthesis Approach to Estimate the Cost of Illness: An Applied Case to the Cost of Depression in Catalonia. Journal of Mental Health, 22, 135-154. https://doi.org/10.3109/09638237.2012.745185

[19] Greenberg, D., Ibrahim, M.I.B.M. and Boncz, I. (2014) What Are the Challenges in Conducting Cost-of-Illness Studies? Value in Health Regional Issues, 4, 115-116. https://doi.org/10.1016/j.vhri.2014.08.003

[20] Jo, C. (2014) Cost-of-Illness Studies: Concepts, Scopes, and Methods. Clinical and Molecular Hepatology, 20, 327-337. https://doi.org/10.3350/cmh.2014.20.4.327

[21] Ng, C.S., Lee, J.Y.C., Toh, M.P.H.S. and Ko, Y. (2014) Cost-of-Illness Studies of Diabetes Mellitus: A Systematic Review. Diabetes Research and Clinical Practice, 105, 151-163. https://doi.org/10.1016/j.diabres.2014.03.020

[22] Sharif, B., Kopec, J., Bansback, N., Rahman, M.M., Flanagan, W.M., Wong, H., Fines, P. and Anis, A. (2015) Projecting the Direct Cost Burden of Osteoarthritis in Canada Using a Microsimulation Model. Osteoarthritis and Cartilage, 23, 1654-1663. https://doi.org/10.1016/j.joca.2015.05.029

[23] Höjvall, J. (2006) A Cost-of-Illness Study of Skin, Soft Tissue, Bone and Lung Infections Caused by Staphylococci. Uppsala University Department of Economics Master Thesis, Uppsala.

[24] Begley, C.E. and Beghi, E. (2002) The Economic Cost of Epilepsy: A Review of the Literature. Epilepsia, 43, 3-9. https://doi.org/10.1046/j.1528-1157.43.s.4.2.x

[25] Van Asselt, A.D.I., Dirksen, C.D., Severens, J.L. and Arntz, A. (2002) Bottom-Up or Top-Down? Impact of Patient Selection on Sot-of-Illness Results. Value in Health, 5, 516-516. https://doi.org/10.1016/S1098-3015(10)61369-1

[26] Koopmanschap, M.A., Redekop, K. and Niessen, L. (2011) Bottom Up versus Top 
Down Cost Estimates for Type 2 Diabetes. ISPOR Fourth Annual European Congress, Cannes, 11-13 Nowember 2001, 411.

[27] Neubauer, G. and Minartz, C. (2007) Pravention von Darmkrebs: Wirksam und Kostengunstig. Die BKK, 6, 265-270.

[28] Sieg, A. and Brenner, H. (2007) Cost-Saving Analysis of Screening Colonoscopy in Germany. Zeitschrift Fur Gastroenterologie, 45, 945-951.

https://doi.org/10.1055/s-2007-963435 